\title{
Octupole based current horn suppression in multistage bunch compression with emittance growth correction
}

\author{
N. Sudar®, Y. Nosochkov, K. Bane, Z. Zhang, and Y. Ding $\odot$ \\ SLAC National Accelerator Laboratory, Menlo Park, California 94025, USA
}

(Received 4 September 2020; accepted 13 November 2020; published 30 November 2020)

\begin{abstract}
High brightness electron beams are critical for $\mathrm{x}$-ray free-electron laser performance. To achieve higher peak current, strong longitudinal compression often produces electron beams with peaks in the head and/or tail of the current profile. These current horns are formed after bunch compression due to nonlinear correlations in the longitudinal phase space and the higher order optics of the compressor. It has been suggested that this higher order compression can be corrected by inserting an octupole magnet near the center of a bunch compressor. However, this scheme provides a correlated transverse kick leading to growth of the projected emittance. We present here a method whereby octupole magnets are inserted into two sequential bunch compressors. By tuning a $\pi$ betatron phase advance between the two octupoles, the correlated transverse kick from the first octupole can be corrected by the second, while providing a cummulative adjustment of the higher order compression.
\end{abstract}

DOI: 10.1103/PhysRevAccelBeams.23.112802

\section{INTRODUCTION}

Advancements in the production of high peak brightness electron beams have revolutionized the field of ultra-fast science through the advent of the $x$-ray Free Electron Laser (FEL). The ability to reach femtosecond level x-ray pulse durations at a growing number of FEL facilities allows for the study of molecular and atomic scale structures as well as femtosecond scale dynamical processes [1]. Meeting the growing demands of the scientific community requires continued improvement in electron beam quality and repetition rate at such facilities.

In order to achieve the electron beam peak current required to drive the FEL interaction, high brightness linear accelerators typically employ multiple 4-dipole chicane bunch compressors. The total compression is limited by non-linear correlations in the electron beam longitudinal phase space. These correlations stem from RF curvature [2], longitudinal space charge (LSC) [3], coherent synchrotron radiation (CSR) [4,5], and resistive wall wakefields [6,7]. Non-linear compression from the second order energy chirp is typically adjusted with a harmonic cavity [8]. Additional methods can be employed to reduce these correlations, [9-16]. However, if left unchecked, higher order compression can lead to the production of horns in the current profile as the head and/or tail of the

Published by the American Physical Society under the terms of the Creative Commons Attribution 4.0 International license. Further distribution of this work must maintain attribution to the author(s) and the published article's title, journal citation, and DOI. electron beam are overcompressed compared to the linear compression in the core of the beam [17-19].

These current horns can produce significant CSR in the bunch compressor causing further distortions in the longitudinal phase space. This correlated longitudinal energy variation can result in a correlated transverse kick leading to growth in the projected emittance, reducing FEL performance as portions of the beam will not be matched ideally to the transverse focusing lattice. Furthermore these current horns can produce significant energy modulation from longitudinal space charge and resistive wall wakefields downstream [20]. In the Linac Coherent Light Source-II (LCLS-II) the electron beam must be transported from the superconducting linac exit through a $2 \mathrm{~km}$ bypass line, requiring control of these collective effects, [21]. As was shown in [22], for high brightness self-amplified spontaneous emission FELs with minimum bandwidth, generating an electron bunch with uniform current profile and flat energy chirp greatly improves FEL performance. This beam quality is even more critical for the performance of self-seeded FELs. Although in a normal conducting linac with a typical beam rate of $100 \mathrm{~Hz}$ or lower, these current horns could be removed with collimation in a dispersive section [22], this is not a viable option for high repetition rate superconducting linacs due to the significant increase in radiated power from beam losses.

It was shown in [23-25], that the growth of current horns can be suppressed by adjusting the higher order compression with an octupole magnet inserted in a bunch compressor. This scheme relies on placing an octupole at a point of significant dispersion, providing a transverse kick correlated with the longitudinal beam coordinate. The octupole kick is 


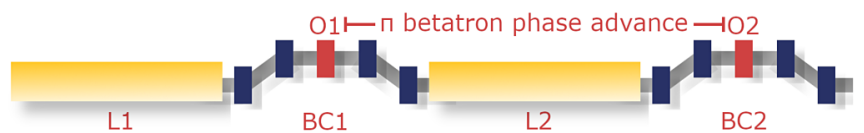

FIG. 1. Illustration of the proposed scheme showing the first linac section (L1), first bunch compressor (BC1) with embedded octupole (O1), second linac section (L2) and second bunch compressor (BC2) with embedded octupole (O2).

then translated into a path length difference through the remainder of the chicane, providing an adjustment of the bunch compressor's third order longitudinal dispersion, $U_{5666}$. However, the correlated transverse kick from the octupole will generally remain imprinted on the beam after the bunch compressor causing significant growth of the projected emittance.

We present here a scheme whereby inserting octupoles in two successive bunch compressors, the projected emittance growth can be corrected. A basic layout of the proposed configuration is shown in Fig. 1. This scheme relies on the cancellation of the octupole kick from the first bunch compressor (BC1) by the octupole in the second one (BC2). Provided that the beam undergoes a $\pi$ betatron phase advance between octupoles, the second octupole kick can be made equal and opposite to the first one while providing an additive contribution to the total $U_{5666}$ of the system. This allows for effective suppression of current horns without significant degradation of the transverse beam quality.

In this paper, we present a study of this scheme using current profile shaping in the LCLS-II superconducting linac as a possible application. Section II gives an analytical description of the longitudinal phase space transformation from an octupole inserted in a bunch compressor. In Sec. III we provide an analytical description of the emittance correction scheme. Section IV shows ELEGANT [26] simulations of a potential configuration for LCLS-II. Section V gives further simulation studies for optimization of the scheme. Section VI provides a discussion of alignment tolerances.

\section{II. $\boldsymbol{U}_{5666}$ from an octupole}

An electron passing through an octupole magnet with negligible vertical offset relative to the magnetic center will receive a horizontal kick depending on its horizontal offset given by [27]:

$$
x^{\prime}=-\frac{B^{\prime \prime \prime}}{6 B \rho} L x^{3} \equiv-\frac{1}{6} K_{3} L x^{3}
$$

Here $L$ is the octupole length and $K_{3}$ is the octupole's geometric strength. Placing an octupole at a point of high dispersion we assume an electron's transverse offset is dominated by its energy deviation from the central energy, $\delta \equiv(\gamma-\langle\gamma\rangle) /\langle\gamma\rangle$. Placing the octupole in the center of a chicane bunch compressor, the transverse offset at the octupole entrance can be expressed by the $R_{16}$ from a simple dogleg:

$$
x_{\mathrm{oct}}=R_{16} \delta \approx-\theta\left(l_{b}+l_{d}\right) \delta .
$$

Here $l_{b}$ is the chicane bend magnet length and $l_{d}$ is the drift length between the 1st and 2nd, and 3rd and 4th chicane magnets, and $\theta$ is the bending angle of the chicane dipoles. The octupole kick then depends on the initial energy offset as:

$$
x_{\mathrm{oct}}^{\prime}=\frac{1}{6} K_{3} L_{0} \theta^{3}\left(l_{b}+l_{d}\right)^{3} \delta^{3} .
$$

The path length difference induced by this kick at the chicane exit is given by the $R_{52}$ from a simple dogleg. The transformation of an electron's longitudinal position, $s$, due to the octupole kick is given by:

$$
\Delta s_{\text {foct }}=R_{52} x_{\mathrm{oct}}^{\prime}=-\frac{1}{6} K_{3} L_{0} \theta^{4}\left(l_{b}+l_{d}\right)^{4} \delta^{3} .
$$

Here note we adopt the convention that the head of the beam points to more negative $s$. The dispersive terms of the chicane including the octupole are given by:

$$
\begin{aligned}
R_{56} & \approx-2 \theta^{2}\left(l_{d}+\frac{2}{3} l_{b}\right) \\
T_{566} & \approx-\frac{3}{2} R_{56} \\
U_{5666} & \approx-\frac{1}{6} K_{3} L \theta^{4}\left(l_{b}+l_{d}\right)^{4}+2 R_{56} .
\end{aligned}
$$

We can approximate the transformation of the initial current profile first considering the evolution of the longitudinal phase space coordinates through the chicane, $\left(s_{i}, \delta_{i}\right) \rightarrow\left(s_{f}, \delta_{f}\right)$. For an electron beam with a non-linear correlated energy chirp described by components, $h_{i}$, this is given by:

$$
\begin{aligned}
& s_{i}=s_{0} \\
& \delta_{i}=\delta_{0}+h_{1} s_{0}+h_{2} s_{0}{ }^{2}+h_{3} s_{0}{ }^{3}+\cdots \\
& s_{f}=s_{i}+R_{56} \delta_{i}+T_{566} \delta_{i}{ }^{2}+U_{5666} \delta_{i}{ }^{3}+\cdots \\
& \delta_{f}=\delta_{i} .
\end{aligned}
$$

In the limit of negligible initial uncorrelated energy spread the transformation of the current profile, $I_{i}$, can be approximated by $I_{f} \approx\left(\left|\frac{\partial s_{f}}{\partial s_{i}}\right|\right)^{-1} I_{i}$. Further discussion of this expression is provided in the Appendix A. The final current profile can be expressed in terms of the linear compression factor, $C_{1}$ and nonlinear compression factors, $c_{11}$ and $c_{12}$ : 


$$
\begin{gathered}
I_{f}\left(s_{f}\right) \approx \frac{C_{1}}{\left|\chi\left(s_{f}\right)\right|} I_{i}\left[s_{i}\left(s_{f}\right)\right] \\
C_{1} \equiv\left(\left.\frac{\partial s_{f}}{\partial s_{i}}\right|_{s_{i}=0}\right)^{-1} \\
c_{11} \equiv C_{1}\left(\left.\frac{\partial^{2} s_{f}}{\partial s_{i}^{2}}\right|_{s_{i}=0}\right) \\
c_{12} \equiv \frac{1}{2} C_{1}\left(\left.\frac{\partial^{3} s_{f}}{\partial s_{i}^{3}}\right|_{s_{i}=0}\right) \\
\chi\left(s_{f}\right)=1+c_{11} s_{i}\left(s_{f}\right)+c_{12} s_{i}\left(s_{f}\right)^{2}+\cdots
\end{gathered}
$$

Current horns will exist in the final current profile approximately where the contribution from nonlinear compression, given by $\chi(s)$, goes to zero. The adjustment of $\chi(s)$ from the octupole allows for a positive nonlinear compression factor along the bunch.
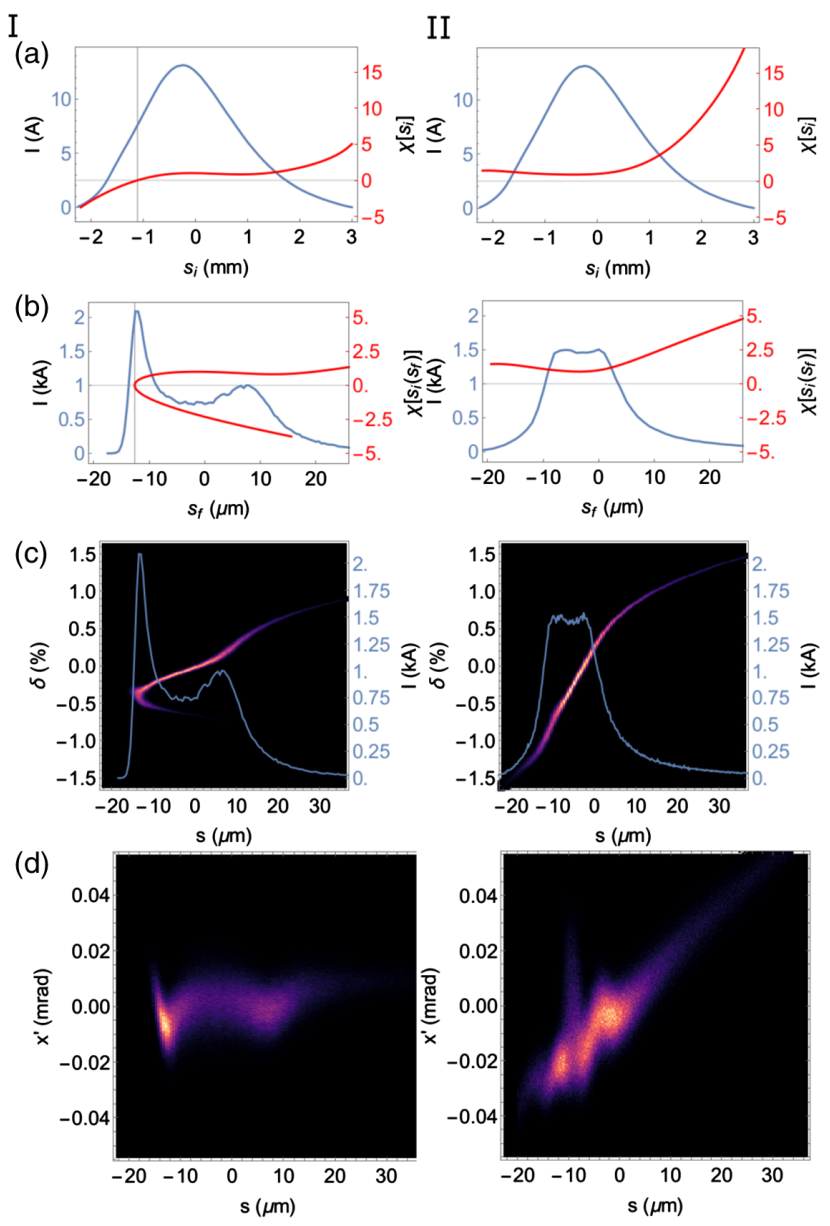

FIG. 2. (I) nominal LCLS-II beam (II) beam with current horns suppressed by single octupole showing: (a) Current profile (blue) and $\chi\left(s_{i}\right)$ (red) at linac entrance. (b) Current profile (blue) and $\chi\left[s_{i}\left(s_{f}\right)\right]$ (red) at BC2 exit. (c) Longitudinal phase space after $\mathrm{BC} 2$ with current profile (blue) for reference. (d) $s$ vs $x^{\prime}$ phase space at BC2 exit. The bunch head is on the left in all plots.
Figure 2 shows an ELEGANT simulation of the nominal compression scheme for the LCLS-II beamline with a strong current horn at the beam head (I) and a hypothetical configuration with an octupole magnet inserted at the center of $\mathrm{BC} 2$ again simulated in ELEGANT (II). Simulations include CSR, LSC and wakefield effects and are done with $5 \times 10^{6}$ macroparticles. In both cases the incoming beam is generated from IMPACT [28,29] simulations of the LCLS-II injector. For the single octupole case, $K_{3} L_{0}=-775 \mathrm{~m}^{-3}$ giving a total $U_{5666}=7.44 \mathrm{~m}$, with remaining electron beam and chicane parameters given in Table 1. For both cases, $\chi(s)$ is shown both as functions of the initial and final longitudinal positions.

Here we see that in the nominal case, $\chi\left(s_{i}\right)$ goes to zero within the initial current profile, leading to horns in the final phase space. The octupole effectively suppresses the current horns and can be used to produce a flat current profile with a factor of two increase in the core peak current.

Figure 2 also shows the $s$ vs $x^{\prime}$ phase space at the exit of the second bunch compressor. From this we see that the correlated octupole kick is preserved at the chicane exit. The normalized projected emittance after BC2 is $\epsilon_{x n}=$ $8.84 \mu \mathrm{m}$ compared with $\epsilon_{x n}=0.4 \mu \mathrm{m}$ for the nominal LCLS-II case.

Assuming a Gaussian energy distribution with RMS energy spread, $\sigma_{\delta}$, the growth of the projected emittance from the octupole kick can be approximated by:

$$
\frac{\epsilon_{x}}{\epsilon_{x 0}} \approx \sqrt{1+\frac{5}{12} \frac{\beta_{x}}{\epsilon_{x 0}}\left(K_{3} L\left(l_{b}+l_{d}\right)^{3} \theta^{3} \sigma_{\delta}^{3}\right)^{2}}
$$

Here $\epsilon_{x 0}$ is the projected geometric emittance at the chicane entrance and $\beta_{x}$ is the electron beam beta function at the octupole. A derivation is provided in Appendix B.

TABLE I. Parameters for LCLS-II double octupole scheme.

\begin{tabular}{lc}
\hline \hline Parameter & Value \\
\hline BC1 e-beam energy $(\mathrm{MeV})$ & 250 \\
e-beam charge $(\mathrm{pC})$ & 100 \\
e-beam chirp @ BC1 entrance $\left(\frac{1}{m}\right)$ & 12.97 \\
emittance @ BC1 entrance $\epsilon_{x, y}(\mu \mathrm{m})$ & $0.3,0.3$ \\
BC1 $R_{56}(\mathrm{~mm})$, bend angle $(\mathrm{mrad})$ & $-47.45,95.66$ \\
BC1 compression factors $C_{1}, c_{11}, c_{12}$ & $2.6,-27.1,2.22 \mathrm{e} 4$ \\
BC1 octupole strength $K_{3}^{(1)} L_{1}\left(m^{-3}\right)$ & -4652.69 \\
BC1 $U_{5666}(\mathrm{~m})$ & 3.254 \\
Beta function @ BC1 octupole $\beta_{x}(\mathrm{~m})$ & 11.05 \\
BC2 e-beam energy $(\mathrm{MeV})$ & 1500 \\
e-beam chirp @ BC2 entrance $\left(\frac{1}{m}\right)$ & 8.34 \\
BC2 $R_{56}(\mathrm{~mm})$, bend angle $(\mathrm{mrad})$ & $-44.93,46.81$ \\
BC2 total compression factors $C_{2}, c_{21}, c_{22}$ & $100,333.8,6.2 \mathrm{e} 5$ \\
BC2 octupole strength $K_{3}^{(2)} L_{2}\left(m^{-3}\right)$ & -511.11 \\
BC2 $U_{5666}(\mathrm{~m})$ & 4.811 \\
Beta function @ BC2 octupole $\beta_{x}(\mathrm{~m})$ & 55.1 \\
core emittance @ BC2 exit $\epsilon_{x n, y n}(\mu \mathrm{m})$ & $0.43,0.41$ \\
\hline \hline
\end{tabular}



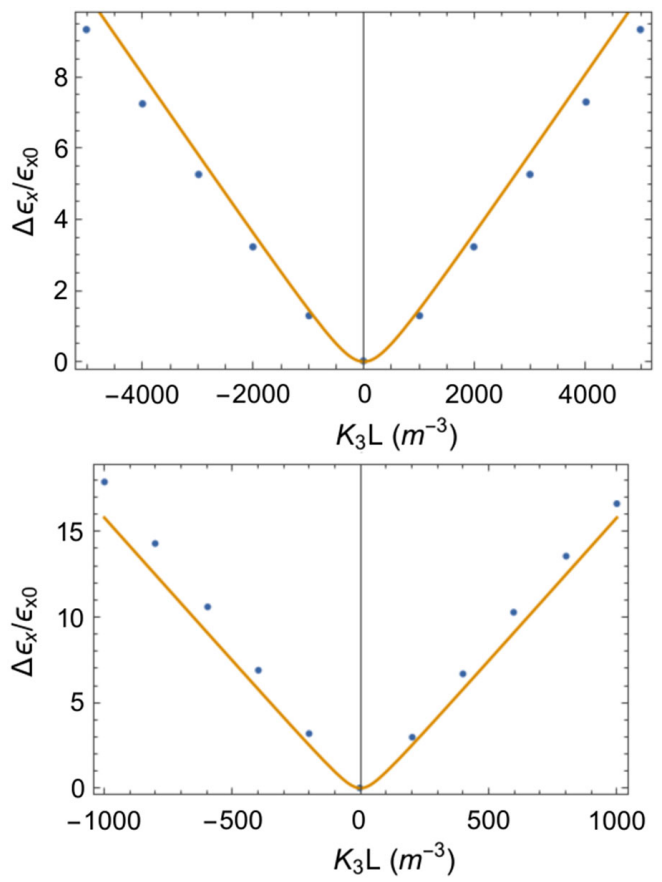

FIG. 3. Change in projected emittance with varying octupole strength from ELEGANT simulations considering an ideal beam with a linear chirp and Gaussian energy distribution (points) and the analytical expression from Eq. (9) (line) for the BC1 octupole (top) and $\mathrm{BC} 2$ octupole (bottom).

Figure 3 shows the emittance growth versus octupole strength from ELEGANT simulations using an idealized Gaussian beam with purely linear correlated chirp. This is done for both $\mathrm{BC} 1$ and $\mathrm{BC} 2$ using electron beam and chicane parameters given in Table 1. Comparison with the analytical estimate from Eq. (9) shows qualitative agreement.

\section{EMITTANCE CORRECTION}

The projected emittance growth problem from the single octupole scheme can be mitigated by splitting the $U_{5666}$ needed to achieve the desired longitudinal shaping between $\mathrm{BC} 1$ and $\mathrm{BC} 2$.

In this double octupole configuration, the correlated kick induced by the first octupole is transported to the second octupole. Tuning the lattice between the two octupoles to provide a $\pi$ betatron phase advance in the bend plane of the chicanes, the $x^{\prime}$ kick from the first octupole is inverted. The second octupole strength can then be set to cancel the first octupole kick while providing additional $U_{5666}$.

We can consider this transformation, writing the $x^{\prime}$ kick at the first octupole in terms of the linear compression factor of the first chicane, $C_{1}$, first octupole strength and length, $K_{3}^{(1)} L_{1}$, chicane bend magnet and drift lengths and bend angle. Writing this kick in terms of the compressed beam coordinate, $s$, after propagation through half of $\mathrm{BC} 1$ gives: $x^{\prime}(s)=\frac{1}{6} K_{3}^{(1)} L_{1}\left(\frac{l_{d 1}+l_{b 1}}{l_{d 1}+\frac{2}{3} l_{b 1}}\right)^{3} \frac{1}{\theta_{1}^{3}}\left(\frac{C_{1}-1}{C_{1}+1}\right)^{3} s^{3}$.

Considering a more general case of $n \pi$ betatron phase advance between the two octupoles, where $n$ is an integer, the $x^{\prime}$ kick due to the first octupole transported to the entrance of the second octupole is given by:

$$
\begin{aligned}
x^{\prime}(s)= & (-1)^{n} \frac{1}{6} K_{3}^{(1)} L_{1} \\
& \times\left(\frac{l_{d 1}+l_{b 1}}{l_{d 1}+\frac{2}{3} l_{b 1}}\right)^{3} \frac{1}{\theta_{1}{ }^{3}}\left(\frac{C_{2}\left(C_{1}-1\right)}{C_{2}+C_{1}}\right)^{3} \sqrt{\frac{\beta_{1} E_{1}}{\beta_{2} E_{2}}} s^{3} .
\end{aligned}
$$

Here $C_{2}$ is the linear compression factor of the second chicane, $\beta_{1}$ and $\beta_{2}$ are the values of the beta function in the bend plane and $E_{1}$ and $E_{2}$ are the values of the central beam energy at the first and second octupoles respectively.

The $x^{\prime}$ kick provided by the second octupole is given by:

$x^{\prime}(s)=\frac{1}{6} K_{3}^{(2)} L_{2}\left(\frac{l_{d 2}+l_{b 2}}{l_{d 2}+\frac{2}{3} l_{b 2}}\right)^{3} \frac{1}{\theta_{2}^{3}}\left(\frac{C_{2}-C_{1}}{C_{2}+C_{1}}\right)^{3} s^{3}$

The net $x^{\prime}$ kick is cancelled when the second octupole kick is equal in magnitude and opposite in sign to the transported kick from the first octupole. In reality, for LCLS-II the sign of the $\mathrm{BC} 2$ chicane dispersion is opposite that of $\mathrm{BC} 1$, requiring an $n 2 \pi$ betatron phase advance for the same cancellation effect. For illustrative purposes we assume throughout that the $\mathrm{BC} 1$ and $\mathrm{BC} 2$ chicane dispersions have the same sign. In the provided example, the phase advance between the two octupoles is $3 \pi$. Further discussion of Eqs. (10)-(12) is provided in Appendix C.

The cancellation requirement gives a condition for the ratio between the two octupole strengths, $\alpha_{K}$ :

$$
\begin{aligned}
\alpha_{K} \equiv \frac{K_{3}^{(2)} L_{2}}{K_{3}^{(1)} L_{1}}= & \left(\frac{\left(l_{d 1}+l_{b 1}\right)\left(l_{d 2}+\frac{2}{3} l_{b 2}\right)}{\left(l_{d 1}+\frac{2}{3} l_{b 1}\right)\left(l_{d 2}+l_{b 2}\right)}\right)^{3}\left(\frac{\theta_{2}}{\theta_{1}}\right)^{3} \\
& \times\left(\frac{C_{2}\left(C_{1}-1\right)}{C_{2}-C_{1}}\right)^{3} \sqrt{\frac{\beta_{1} E_{1}}{\beta_{2} E_{2}}}
\end{aligned}
$$

For a given total $U_{5666} \equiv U_{\text {tot }}$ we can split the octupole strengths according to Eq. (13) and (5).

$K_{3}^{(1)} L_{1}=-\frac{6 U_{\text {tot }}+24 \theta_{1}^{2}\left(l_{d 1}+\frac{2}{3} l_{b 1}\right)+24 \theta_{2}^{2}\left(l_{d 2}+\frac{2}{3} l_{b 2}\right)}{\theta_{1}{ }^{4}\left(l_{b 1}+l_{d 1}\right)^{4}+\alpha_{K} \theta_{2}{ }^{4}\left(l_{b 2}+l_{d 2}\right)^{4}}$.

Figure 4, shows the octupole kick from the first (a) and second (b) octupoles, the first octupole kick transported to the entrance of the second octupole (c) and the kick 

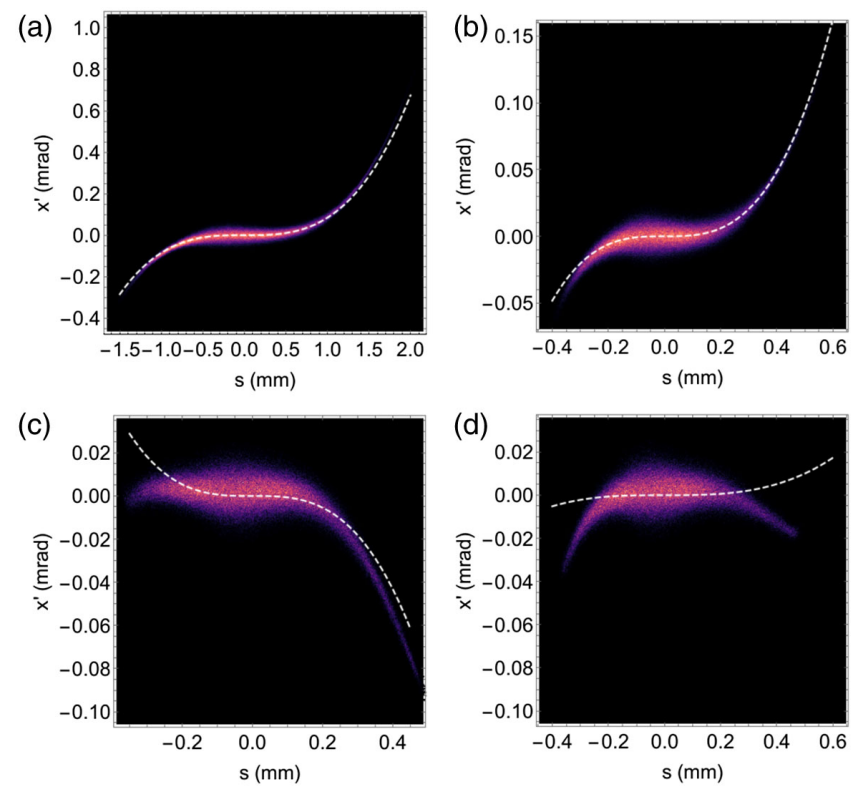

FIG. 4. From ELEGANT simulations: (a) Correlated kick from the first octupole ( $s$ vs $x^{\prime}$ ) with the analytical estimate from Eq. (10) (white dashed), (b) Kick from the second octupole with first octupole off with the analytical estimate from Eq. (12) (white dashed), (c) Kick from the first octupole transported to the second octupole entrance with the analytical estimate from Eq. (11), (d) Combined kick from the first and second octupoles with octupole strengths chosen to minimize projected emittance of the core of the beam. The bunch head is on the left in all plots.

cancellation at the second octupole exit (d) from ELEGANT simulations of the LCLS-II linac configuration described in Sec. IV. The analytical estimates from Eqs. (10)-(12) are shown for comparison. The discrepancy between the analytical expressions and simulations can be attributed to second order chromatic focusing effects in the quadrupoles between $\mathrm{BC} 1$ and $\mathrm{BC} 2$. The $T_{161}, T_{162}, T_{261}$, and $T_{262}$ associated with this transport produce an additional approximately quadratic correlated $\mathrm{x}$ and $\mathrm{x}$ ' kick, which is not included in the analytical expressions. This is highlighted in particular by the comparison between Eq. (11) and the transported first octupole kick from simulation, Fig. 4(c). Additional discussion of the emittance correction scheme can be found in [30].

\section{LCLS-II DOUBLE OCTUPOLE CONFIGURATION}

From the single octupole case shown in Sec. II, an initial choice for the $\mathrm{BC} 1$ and $\mathrm{BC} 2$ octupole strengths is found by splitting the total $U_{5666}$ between the two chicanes according to Eq. (14). Some adjustment of the total $U_{5666}$ must be made to obtain an identical current profile due to the change in higher order compression at BC1. Figure 5, shows the longitudinal phase space at the exit of $\mathrm{BC} 2$ and undulator entrance with the double octupole configuration. The $s$ vs $x^{\prime}$ phase space at the $\mathrm{BC} 2$ exit shows the correction of the
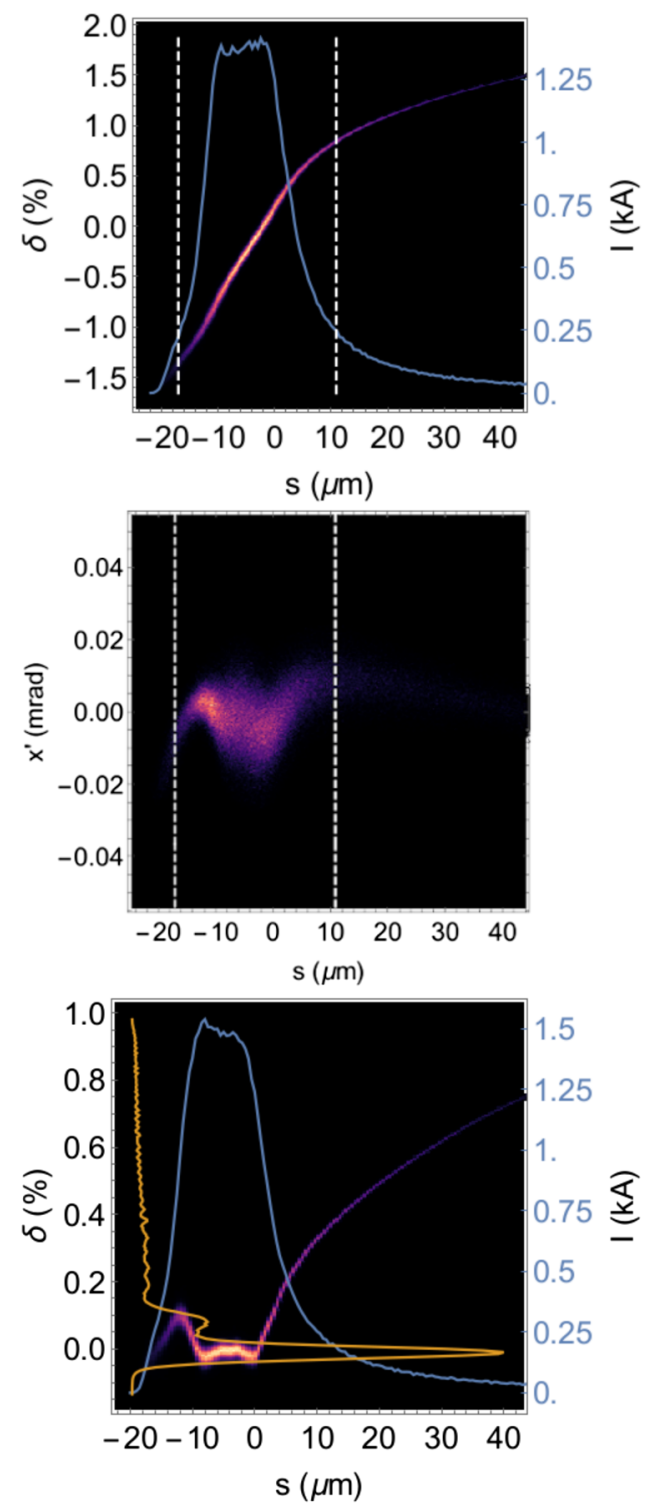

FIG. 5. Top: the longitudinal phase space at the exit of $\mathrm{BC} 2$, with current profile (blue), the white dashed lines define the core of the beam. Middle: $s$ vs $x^{\prime}$ at the exit of BC2. Bottom: longitudinal phase space at the undulator entrance with current profile (blue) and energy lineout (yellow). The bunch head is on the left in all plots.

octupole kick. The residual chirp on the core part of the bunch after the final acceleration section is removed by the resistive wall wakefield and longitudinal space charge in the $\sim 2 \mathrm{~km}$ bypass line. The additional non-linearities observed in the head and tail of the final longitudinal phase space mainly come from the initial injector beam, with additional contributions from the linac wakefields. Parameters are given in Table I.

We define the core of the beam as the region within $>10 \%$ of the peak current, with this region shown by the white dashed lines in Fig. 5. The ratio between octupole strengths can be optimized further in simulations to 


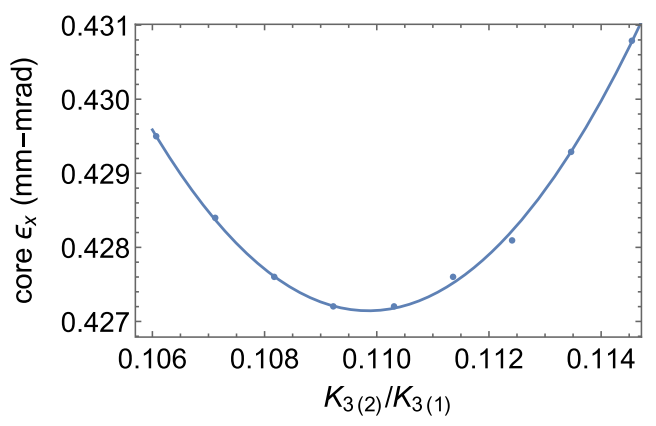

FIG. 6. The normalized core projected emittance with varying octupole strength ratios maintaining the total $U_{5666}$ from ELEGANT simulations (points) with quadratic fit (line).

minimize the projected emittance over the core of the beam, correcting for effects not considered in the provided analysis, i.e., higher-order dispersion, CSR, LSC, and chromatic focusing.

Figure 6, shows the emittance growth in the core of the beam when varying the octupole strength ratio while maintaining the total $U_{5666}$ from ELEGANT simulations. This gives an optimal ratio $\alpha_{K}=0.1099$ showing good agreement with the analytical estimate from Eq. (13), which gives $\alpha_{K}=0.098$. After BC2 the normalized core projected emittance is $\epsilon_{x n}=0.43 \mu \mathrm{m}$ compared with $\epsilon_{x n}=0.31 \mu \mathrm{m}$ at the BC1 entrance and $\epsilon_{x n}=3.1 \mu \mathrm{m}$ from the single octupole case. The remnant increase in projected emittance can be again attributed to second order focusing between octupoles. Varying $\alpha_{K}$ by $\pm 5 \%$ shows negligible increase in the projected emittance. In the provided LCLS-II case, for an octupole length, $L=0.2 \mathrm{~m}$, the quoted octupole strengths correspond to modest pole tip fields of $1.4 \mathrm{kG}$ and $0.33 \mathrm{kG}$ with an aperture of $70 \mathrm{~mm}$ and $50 \mathrm{~mm}$ respectively, with both values larger than the beam clearance requirement of the corresponding bunch compressor.

Figure 7 shows the beta function, $\beta_{x}$, dispersion, $\eta_{x}$, and betatron phase advance, $\phi_{\beta x}$ in the bend plane of $\mathrm{BC} 1$ and $\mathrm{BC} 2$. These quantities are plotted from the entrance of $\mathrm{BC} 1$ $(z=0)$ to the exit of $\mathrm{BC} 2$ from ELEGANT simulations, with $\phi_{\beta x}=0$ at the first octupole.
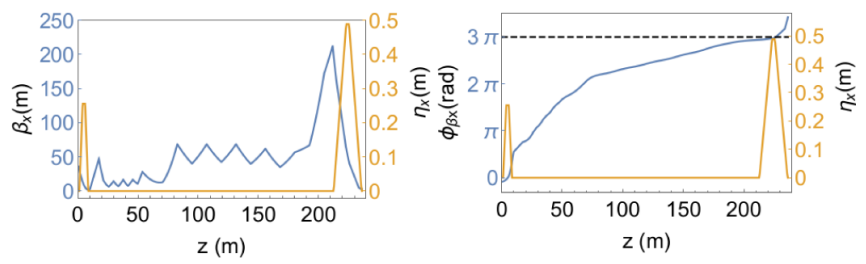

FIG. 7. Left: the beta function, $\beta_{x}$, (blue) and dispersion in the bend plane, $\eta_{x}$, (yellow). Right: the betatron phase advance, $\phi_{\beta x}$, (blue) and dispersion in the bend plane, $\eta_{x}$ (yellow) with the dashed line showing the phase advance at the second octupole. Here quantities are taken from ELEGANT simulations and are plotted from the entrance of $\mathrm{BC} 1(z=0)$ to the exit of $\mathrm{BC} 2$.

\section{LINAC CONFIGURATION CONSIDERATIONS}

In choosing a linac configuration for the double octupole scheme it is advantageous to minimize the emittance growth after the first bunch compressor. In the case of the LCLS-II beamline, there are halo collimators located downstream of $\mathrm{BC} 1$ to help satisfy the beam stay-clear requirement. For some configurations, we observe particle losses of a few percent due to the large angular kick at the bunch tail. If the particle loss is above the collimator loss threshold, we have to either lower the beam rate or use a different configuration with weaker octupole strength.

We can gain some insight toward an optimal linac configuration using Eq. (9) to estimate the BC1 emittance growth for varying linac and chicane parameters that give approximately the same final current profile. This is done by constraining the total linear compression, $\mathrm{BC} 1$ and $\mathrm{BC} 2$ nonlinear compression factors, Eq. (7), the electron beam energy at $\mathrm{BC}$, and the emittance cancellation condition, Eq. (13). With current horn suppression from the double octupole scheme, the energy modulation from linac wakefields dominates over LSC and CSR. For changes in the BC1 compression, the linac wakefield between $\mathrm{BC} 1$ and $\mathrm{BC} 2$ can be approximately scaled by the $\mathrm{BC} 1$ linear compression factor.

Figure 8 shows the estimated projected emittance growth after $\mathrm{BC} 1$ when varying the $\mathrm{BC} 1 R_{56}$, electron beam linear chirp at the entrance of $\mathrm{BC} 1$, and the electron beam energy at $\mathrm{BC} 1$. Here we only consider linac configurations within the specifications of the LCLS-II linac. From this we see a general trend that the $\mathrm{BC} 1$ emittance growth decreases with increasing chirp and decreasing $R_{56}$. Furthermore, for the same chirp and $R_{56}$ the emittance growth decreases with increasing electron beam energy at $\mathrm{BC} 1$. The parameters for the provided LCLS-II case are chosen to optimize the final phase space at the undulator entrance. Notably, Fig. 8 illustrates that the desired longitudinal beam shaping can be achieved over a wide range of linac parameters. A discussion of other possible LCLS-II linac configurations is given in [31].

As previously stated, the remnant emittance growth is dominated by second order chromatic focusing. Therefore, the decreased correlated kick after BC1 and increased chirp will approximately cancel, and not lead to a reduction of the projected emittance growth after BC2. For instance, the linac configuration corresponding to $E=270 \mathrm{MeV}$, $R_{56}=35 \mathrm{~mm}$ and $h=15.5 \mathrm{~m}^{-1}$ gives a final emittance of $0.4375 \mathrm{~mm}$-mrad compared with $0.43 \mathrm{~mm}$-mrad for the presented case. However, for this case the required octupole strengths at $\mathrm{BC} 1$ and $\mathrm{BC} 2$ are reduced to $K_{3}^{(1)} L_{1}=-3607.1$ and $K_{3}^{(2)} L_{2}=-315.6$, respectively. This reduction of the octupole strength requirement could be advantageous when considering practical application. 

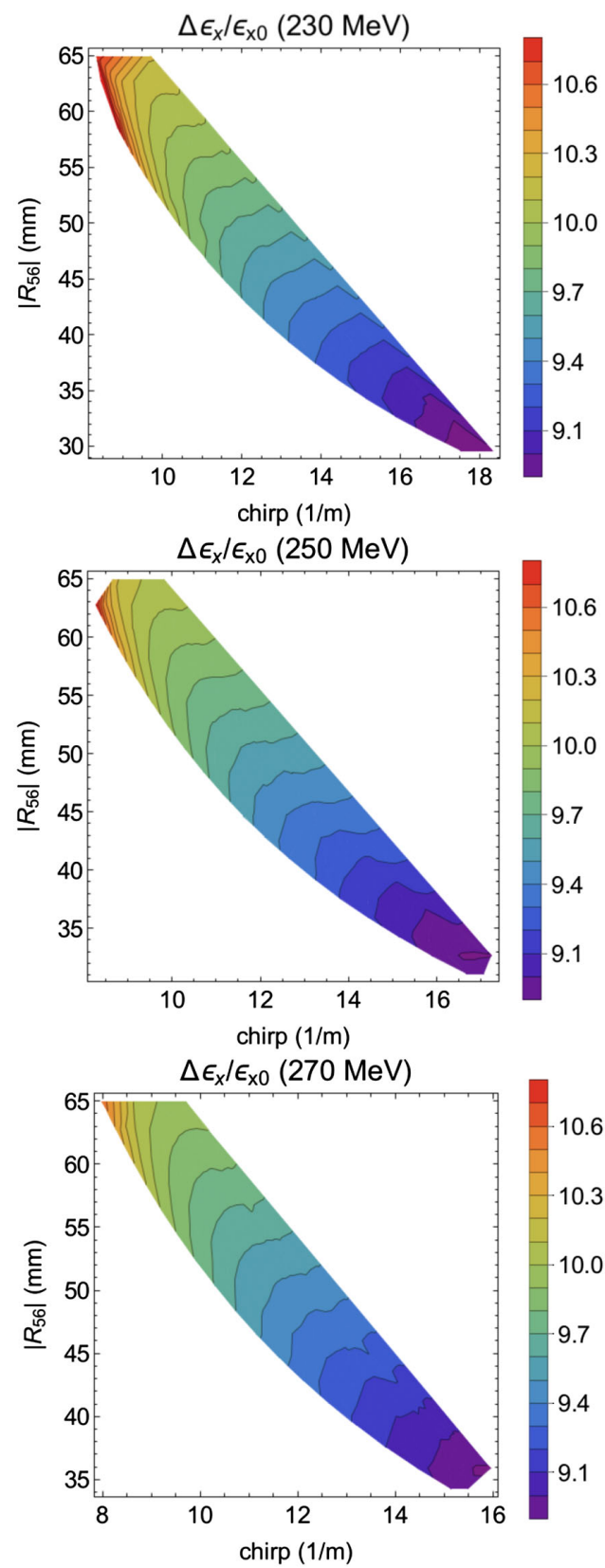

FIG. 8. The estimated emittance growth after $\mathrm{BC} 1$, with varying $\mathrm{BC} 1 R_{56}$ and linear chirp at the $\mathrm{BC} 1$ entrance for BC1 energy $230 \mathrm{MeV}$ (top), $250 \mathrm{MeV}$ (middle) and $270 \mathrm{MeV}$ (bottom).

\section{ALIGNMENT TOLERANCE}

Transverse misalignment of the octupole will result in a normal and skew sextupole like kicks. To see this we can write the octupole field shifted by $\Delta x$ and $\Delta y$ :

$$
\begin{aligned}
& B_{y}=\frac{B^{\prime \prime \prime}}{6}\left[(x-\Delta x)^{3}-3(x-\Delta x)(y-\Delta y)^{2}\right] \\
& B_{x}=\frac{B^{\prime \prime \prime}}{6}\left[3(x-\Delta x)^{2}(y-\Delta y)-(y-\Delta y)^{3}\right] .
\end{aligned}
$$

Assuming this misalignment is small compared to the dispersion and the electron beam's vertical offset relative to the magnetic center of the octupole is dominated by the vertical shift, we can write the field keeping the lowest order terms in $\Delta x$ and $\Delta y$ :

$$
\begin{aligned}
& B_{y}=\frac{B^{\prime \prime \prime}}{6}\left(x^{3}-3 x^{2} \Delta x\right) \\
& B_{x}=-\frac{B^{\prime \prime \prime}}{2}\left(x^{2} \Delta y\right) .
\end{aligned}
$$

For the octupole inserted in the chicane, the horizontal offset at the octupole is dominated by dispersion. The horizontal and vertical kick induced by the shifted octupole is then given by:

$$
\begin{aligned}
x^{\prime} & =\frac{1}{6} K_{3} L \theta^{3}\left(l_{b}+l_{d}\right)^{3} \delta^{3}-\frac{1}{2} K_{3} L \theta^{2}\left(l_{b}+l_{d}\right)^{2} \delta^{2} \Delta x \\
y^{\prime} & =-\frac{1}{2} K_{3} L \theta^{2}\left(l_{b}+l_{d}\right)^{2} \delta^{2} \Delta y .
\end{aligned}
$$

The additional sextupole kick will remain imprinted on the transverse phase space causing additional emittance growth. Provided the octupole kicks are cancelled, the emittance growth at the exit of $\mathrm{BC} 2$ from a misaligned octupole is approximately given by:

$$
\begin{aligned}
\frac{\Delta \epsilon_{x}}{\epsilon_{x}} & \approx \frac{3}{8} \frac{\beta_{x}}{\epsilon_{x}}\left(K_{3} L \theta^{2}\left(l_{b}+l_{d}\right)^{2} \sigma_{\delta}^{2} \Delta x\right)^{2} \\
\frac{\Delta \epsilon_{y}}{\epsilon_{y}} & \approx \frac{3}{8} \frac{\beta_{y}}{\epsilon_{y}}\left(K_{3} L \theta^{2}\left(l_{b}+l_{d}\right)^{2} \sigma_{\delta}^{2} \Delta y\right)^{2}
\end{aligned}
$$

Here $l_{b}, l_{d}, \theta, K_{3}$ and $L$ refer to the $\mathrm{BC} 1$ or $\mathrm{BC} 2$ chicane and octupole parameters, $\beta_{x, y}$ and $\epsilon_{x, y}$ are the beta function and geometric emittance at the octupole, and $\sigma_{\delta}$ is the RMS energy spread at the chicane entrance. This expression gives $10 \% \mathrm{x}$ emittance growth for $\pm 100 \mu \mathrm{m}$ offset of the $\mathrm{BC} 1$ and $\mathrm{BC} 2$ octupoles and $10 \%$ y emittance growth for $\pm 50 \mu \mathrm{m}$ offset of the $\mathrm{BC} 1$ octupole and $\pm 100 \mu \mathrm{m}$ of the $\mathrm{BC} 2$ octupole.

These alignment tolerances are relaxed when we consider emittance growth in the core of the beam including the additional emittance growth observed in ELEGANT simulations. Figure 9, shows the proportional increase in normalized emittance, with varying $\mathrm{x}$ and $\mathrm{y}$-offset of the $\mathrm{BC} 1$ and $\mathrm{BC} 2$ octupoles in ELEGANT. From this we see $10 \%$ y-emittance growth for $\pm 150 \mu \mathrm{m}$ offset of the BC1 octupole and $\pm 200 \mu \mathrm{m}$ for the BC2 octupole and $10 \%$ $\mathrm{x}$-emittance growth for $\pm 200 \mu \mathrm{m}$ offset of the $\mathrm{BC} 1$ and BC2 octupoles. In Fig. 9(a), we observe that the remnant emittance growth caused by 2 nd order chromatic focusing is reduced by the sextupole kick from $\mathrm{x}$-offset of the octupoles. Furthermore, similarly to the octupole emittance correction method, the $\mathrm{x}$-emittance growth due to the $\mathrm{BC} 1$ sextupole kick can be corrected by the sextupole kick from 
(a)

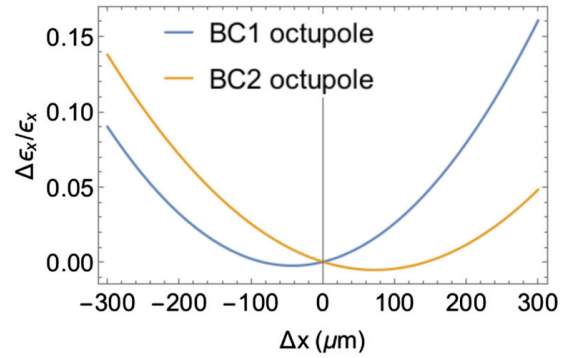

(b)

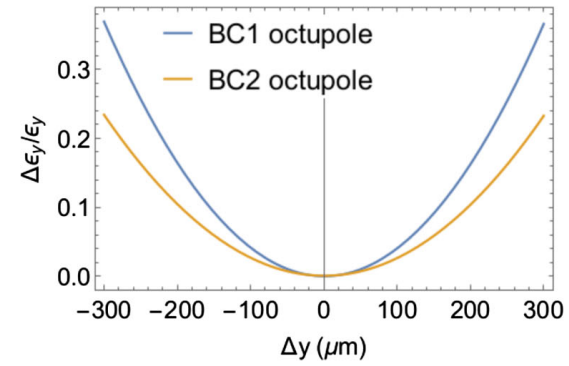

(c)
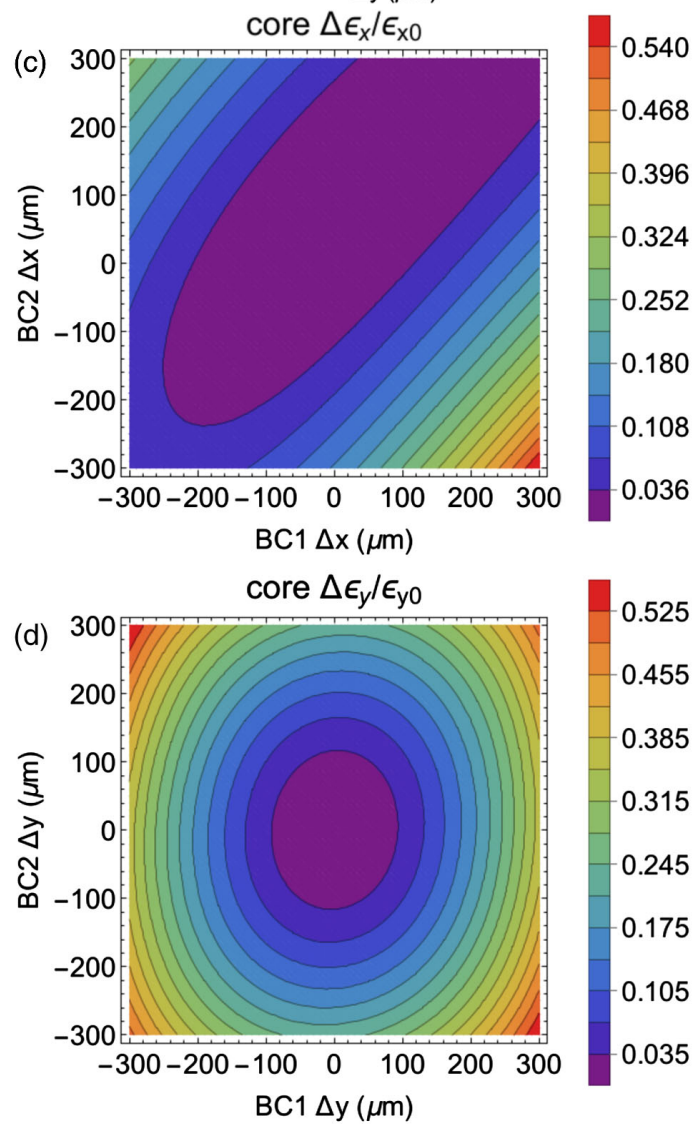

FIG. 9. (a) The proportional increase in the $\mathrm{x}$ emittance in the core of the beam at the exit of $\mathrm{BC} 2$, with varying $\mathrm{BC} 1$ octupole $\mathrm{x}$-offset (blue) and $\mathrm{BC} 2$ octupole x-offset (yellow) (b) The proportional increase in the $\mathrm{y}$ emittance in the core of the beam at the exit of $\mathrm{BC} 2$, with varying $\mathrm{BC} 1$ octupole y-offset (blue) and $\mathrm{BC} 2$ octupole y-offset (yellow). (c) The proportional increase in the $\mathrm{x}$ emittance in the core of the beam at the exit of $\mathrm{BC} 2$, with varying both $\mathrm{BC} 1$ and $\mathrm{BC} 2 \mathrm{x}$-offset. (d) The proportional increase in the $\mathrm{x}$ emittance in the core of the beam at the exit of $\mathrm{BC} 2$, with varying both $\mathrm{BC} 1$ and $\mathrm{BC} 2$-offset. the $\mathrm{BC} 2$ octupole. For our case, with $3 \pi$ betatron phase advance between octupoles, this correction occurs for $\mathrm{x}$-offsets in the same direction. This effect can possibly be utilized to reduce the final emittance growth by adding motion control of the octupole magnets.

\section{CONCLUSION}

The double octupole scheme demonstrates effective suppression of current horns by adjusting the higher order compression of a linac with multistage bunch compression. This allows for the generation of a flat current profile with an increase in the achievable peak current and preservation of the transverse beam quality. This method can be incorporated in most existing high brightness linacs, and could be improved upon if considered in the initial design of such a facility.

\section{ACKNOWLEDGMENTS}

The authors would like to thank Agostino Marinelli, Tessa Charles, and Paul Emma for useful discussion. This work was supported by U.S. Department of Energy Contract No. DE-AC02-76SF00515.

\section{APPENDIX A: EQ. (8) DERIVATION}

In the limit of small uncorrelated energy spread we can consider an initial distribution function with current profile described by the function $I_{i}\left(s_{0}\right)$ and a delta function energy distribution:

$$
F\left[s_{0}, \delta_{0}\right]=I_{i}\left[s_{0}\right] \delta\left(\delta_{0}\right) .
$$

After acquiring a nonlinear chirp the longitudinal position and energy detuning, $\left(s_{i}, \delta_{i}\right)$, are given by:

$$
\begin{aligned}
& s_{i}=s_{0} \\
& \delta_{i}=\delta_{0}+h_{1} s_{0}+h_{2} s_{0}{ }^{2}+h_{3} s_{0}{ }^{3} .
\end{aligned}
$$

After nonlinear compression the longitudinal phase space is transformed as:

$$
\begin{aligned}
& s_{f}=s_{i}+R_{56} \delta_{i}+T_{566} \delta_{i}{ }^{2}+U_{5666} \delta_{i}{ }^{3} \\
& \delta_{f}=\delta_{i} .
\end{aligned}
$$

The distribution function can be written in terms of the transformed phase space variables:

$$
F_{1}\left[s_{f}, \delta_{f}\right]=I_{i}\left[s_{0}\left[s_{f}, \delta_{f}\right]\right] \delta\left(\delta_{0}\left[s_{f}, \delta_{f}\right]\right) .
$$

With $s_{0}\left[s_{f}, \delta_{f}\right]$ and $\delta_{0}\left[s_{f}, \delta_{f}\right]$ given by: 


$$
\begin{aligned}
& s_{0}\left[s_{f}, \delta_{f}\right]=s_{f}-R_{56} \delta_{f}-T_{566} \delta_{f}^{2}-U_{5666} \delta_{f}^{3} \\
& \delta_{0}\left[s_{f}, \delta_{f}\right]=\delta_{f}-h_{1} s_{0}\left[s_{f}, \delta_{f}\right]-h_{2} s_{0}\left[s_{f}, \delta_{f}\right]^{2}-h_{3} s_{0}\left[s_{f}, \delta_{f}\right]^{3} .
\end{aligned}
$$

Integrating over energy gives the final current profile:

$$
I_{f}\left[s_{f}\right]=\int_{-\infty}^{\infty} d \delta_{f} I_{i}\left[s_{0}\left[s_{f}, \delta_{f}\right]\right] \delta\left(\delta_{0}\left[s_{f}, \delta_{f}\right]\right) .
$$

For some position, $s_{f}$, the solution is written in terms of a sum over the roots of $\delta_{0}\left[s_{f}, \delta_{f 0^{(j)}}\right]=0$ :

$$
\begin{aligned}
I_{f}\left[s_{f}\right]= & \sum_{j} \frac{I_{i}\left[s_{0}\left[s_{f}, \delta_{f 0}^{(j)}\right]\right]}{\left|\frac{\partial}{\partial \delta_{f}} \delta_{0}\left[s_{f}, \delta_{f 0}^{(j)}\right]\right|}=\sum_{j} I_{i}\left[s_{0}\left[s_{f}, \delta_{f 0}^{(j)}\right]\right] \\
& \times\left(\mid 1+\left(h_{1}+2 h_{2} s_{0}\left[s_{f}, \delta_{f 0}^{(j)}\right]+3 h_{3} s_{0}\left[s_{f}, \delta_{f 0}^{(j)}\right]^{2}\right)\right. \\
& \left.\times\left(R_{56}+2 T_{566} \delta_{f 0}^{(j)}+3 U_{5666} \delta_{f 0}^{(j) 2}\right) \mid\right)^{-1}
\end{aligned}
$$

The roots, $\delta_{f 0}^{(j)}$, satisfy:

$\delta_{f 0}^{(j)}-h_{1} s_{0}\left[s_{f}, \delta_{f 0}^{(j)}\right]-h_{2} s_{0}\left[s_{f}, \delta_{f 0}^{(j)}\right]^{2}-h_{3} s_{0}\left[s_{f}, \delta_{f 0}^{(j)}\right]^{3}=0$.

For no initial energy offset, $\delta_{0}=0, s_{f}$ depends only on the initial beam coordinate, $s_{0}$, giving:

$$
\delta_{f 0}^{(j)}=h_{1} s_{0}^{(j)}\left(s_{f}\right)+h_{2} s_{0}^{(j)}\left(s_{f}\right)^{2}+h_{3} s_{0}^{(j)}\left(s_{f}\right)^{3} .
$$

Here the $j$ index refers to the roots of $s_{f}-s_{f}\left[s_{0}\right]=0$. Substituting Eq. (A9) into Eq. (A7) gives:

$$
I_{f}\left[s_{f}\right]=\sum_{j} \frac{I_{i}\left[s_{0}^{(j)}\left(s_{f}\right)\right]}{\left|1+\frac{\partial \delta_{f 0}^{(j)}}{\partial s_{0}}\left(R_{56}+2 T_{566} \delta_{f 0}^{(j)}+3 U_{5666} \delta_{f 0}^{(j) 2}\right)\right|} .
$$

The denominator of Eq. (A10) can be conveniently expressed by, $\frac{\partial s_{f}}{\partial s_{i}}$, evaluated at $s_{i}^{(j)}\left(s_{f}\right)$ :

$$
I_{f}\left[s_{f}\right]=\sum_{j} \frac{I_{i}\left[s_{i}^{(j)}\left(s_{f}\right)\right]}{\left|\frac{\partial s_{f}}{\partial s_{i}}\left[s_{i}^{(j)}\left(s_{f}\right)\right]\right|} .
$$

\section{APPENDIX B: EQ. (9) DERIVATION}

The transformation of the transverse phase space from the entrance to the exit of the bunch compressor with octupole in the center is given in terms of the initial phase space coordinates $\left(x_{0}, x_{0}^{\prime}\right)$ :

$$
\begin{aligned}
& x_{f} \approx x_{0}+2 L x_{0}^{\prime}+L \frac{1}{6} K_{3} L_{0} \eta^{3} \delta_{0}{ }^{3} \\
& x_{f}^{\prime} \approx x_{0}^{\prime}+\frac{1}{6} K_{3} L_{0} \eta^{3} \delta_{0}^{3} .
\end{aligned}
$$

Here $\eta$ is the transverse dispersion at the octupole. For an octupole at the center of a bunch compressor $\eta=\left(l_{b}+l_{d}\right) \theta$, with $l_{b}$ is the magnet length, $l_{d}$ is the drift length between the 1 st and 2 nd and 3 rd and 4 th dipoles, $L$ is half the full length of the chicane, $L_{0}$ is the length of the octupole, $K_{3}$ is the octupole strength and $\theta$ is the chicane bend angle. Defining $x_{K} \equiv \frac{1}{6} K_{3} L_{0} \eta^{3} \delta_{0}{ }^{3}$, the emittance at the chicane exit is given by:

$$
\begin{gathered}
\epsilon^{2}=\left\langle x_{f}{ }^{2}\right\rangle\left\langle x_{f}^{\prime 2}\right\rangle-\left\langle x_{f} x_{f}^{\prime}\right\rangle^{2} \\
\epsilon^{2}=\left(\left\langle\left(x_{0}+2 L x_{0}^{\prime}\right)^{2}\right\rangle+2 L\left\langle\left(x_{0}+2 L x_{0}^{\prime}\right) x_{K}\right\rangle+L^{2}\left\langle x_{K}{ }^{2}\right\rangle\right) \\
\times\left(\left\langle x_{0}^{\prime 2}\right\rangle+2\left\langle x_{0}^{\prime} x_{K}\right\rangle+\left\langle x_{K}^{2}\right\rangle\right)-\left(\left\langle\left(x_{0}+2 L x_{0}^{\prime}\right) x_{0}^{\prime}\right\rangle\right. \\
\left.+\left\langle\left(x_{0}+2 L x_{0}^{\prime}\right) x_{K}\right\rangle+L\left\langle x_{0}^{\prime} x_{K}\right\rangle+L\left\langle x_{K}^{2}\right\rangle\right)^{2} .
\end{gathered}
$$

The octupole kick, $x_{K}$, is approximately uncorrelated with $x_{0}$ and $x_{0}^{\prime}$, giving $\left\langle x_{0} x_{K}\right\rangle \sim\left\langle x_{0}^{\prime} x_{K}\right\rangle \sim 0$. Equation (B3) can then be written in terms of the emittance growth from the octupole and the initial geometric emittance:

$$
\begin{aligned}
\epsilon^{2}= & \left\langle\left(x_{0}+2 L x_{0}^{\prime}\right)^{2}\right\rangle\left\langle x_{0}^{\prime 2}\right\rangle-\left\langle\left(x_{0}+2 L x_{0}^{\prime}\right) x_{0}^{\prime}\right\rangle^{2} \\
& +L^{2}\left\langle x_{0}^{\prime 2}\right\rangle\left\langle x_{K}{ }^{2}\right\rangle+\left\langle\left(x_{0}+2 L x_{0}^{\prime}\right)^{2}\right\rangle\left\langle x_{K}{ }^{2}\right\rangle \\
& -2 L\left\langle\left(x_{0}+2 L x_{0}^{\prime}\right) x_{0}^{\prime}\right\rangle\left\langle x_{K}{ }^{2}\right\rangle \\
\epsilon^{2}= & \epsilon_{0}{ }^{2}+\left\langle\left(x_{0}+2 L x_{0}^{\prime}\right)^{2}\right\rangle\left\langle\left(\frac{1}{6} K_{3} L_{0} \eta^{3} \delta_{0}{ }^{3}\right)^{2}\right\rangle .
\end{aligned}
$$

The emittance growth is given in terms of the beta function at the octupole, with $\epsilon_{0} \beta_{x}=\left\langle\left(x_{0}+L x_{0}^{\prime}\right)^{2}\right\rangle$ :

$$
\frac{\epsilon}{\epsilon_{0}}=\sqrt{1+\frac{1}{36} \frac{\beta_{x}}{\epsilon_{0}}\left(K_{3} L_{0}\right)^{2} \eta^{6}\left\langle\delta_{0}{ }^{6}\right\rangle}
$$

Assuming a Gaussian energy distribution at the chicane entrance we find $\left\langle\delta_{0}{ }^{6}\right\rangle=15 \sigma_{\delta}$. Inserting the dispersion from Eq. (2) gives the expression for the emittance growth, given by Eq. (9) in the text:

$$
\frac{\epsilon}{\epsilon_{0}}=\sqrt{1+\frac{5}{12} \frac{\beta_{x}}{\epsilon_{0}}\left(K_{3} L_{0}\left(l_{b}+l_{d}\right)^{3} \theta^{3} \sigma_{\delta}^{3}\right)^{2}}
$$

\section{APPENDIX C: EQS. (10)-(12) DERIVATION}

In order to study the emittance correction scheme, we first consider a beam with a linear chirp, $h_{11}$ at the $\mathrm{BC} 1$ chicane entrance. The longitudinal position and energy detuning, $\left(s_{1}, \delta_{1}\right)$ can be expressed in terms of the initial longitudinal position, $s_{0}$ : 


$$
\begin{aligned}
& \delta_{1}=h_{11} s_{0} \\
& s_{1}=s_{0} .
\end{aligned}
$$

Assuming the octupole is located at the halfway point of the $\mathrm{BC} 1$ chicane, the longitudinal phase space coordinates at the octupole entrance are given in terms of the total $\mathrm{BC} 1$ $R_{56}$ :

$$
\begin{aligned}
& \delta_{2}=h_{11} s_{0} \\
& s_{2}=s_{1}+\frac{R_{56}^{(1)}}{2} \delta_{1}=s_{0}+\frac{R_{56}^{(1)}}{2} h_{11} s_{0} .
\end{aligned}
$$

After the octupole, the transverse coordinates in the bend plane of the chicane, $\left(x_{2}, x_{2}^{\prime}\right)$, are given in terms of the dispersion at the octupole entrance, $\eta_{1}$, and the octupole kick:

$$
\begin{aligned}
& x_{2}=\eta_{1} \delta_{1}=\eta_{1} h_{11} s_{0} \\
& x_{2}^{\prime}=\frac{1}{6} K_{3}^{(1)} L_{1} x_{2}{ }^{3} .
\end{aligned}
$$

At the chicane exit, the longitudinal phase space coordinates can be expressed in terms of the $\mathrm{BC} 1$ linear compression factor, $C_{1}$ :

$$
\begin{aligned}
\delta_{3} & =h_{11} s_{0} \\
s_{3} & =s_{0}+R_{56}^{(1)} \delta_{2}=s_{0}+R_{56}^{(1)} h_{11} s_{0}=\frac{s_{0}}{C_{1}} \\
C_{1} & =\frac{1}{1+h_{11} R_{56}^{(1)}} .
\end{aligned}
$$

After transport through an accelerating section, the beam acquires an additional linear chirp component, $h_{21}$. The longitudinal phase space coordinates are given in terms of the beam energy at $\mathrm{BC} 1, E_{1}$, and $\mathrm{BC} 2, E_{2}$, with $R_{66}=\frac{E_{1}}{E_{2}}$ :

$$
\begin{aligned}
& \delta_{4}=h_{11} R_{66} s_{0}+h_{21} s_{3}=h_{11} R_{66} s_{0}+\frac{h_{21}}{C_{1}} s_{0} \\
& s_{4}=\frac{s_{0}}{C_{1}} .
\end{aligned}
$$

Again, assuming the second octupole is located at the halfway point of the $\mathrm{BC} 2$ chicane, the longitudinal phase space coordinates at the octupole entrance are given in terms of the total $\mathrm{BC} 2 R_{56}$ :

$\delta_{5}=h_{11} R_{66} s_{0}+\frac{h_{21}}{C_{1}} s_{0}$

$s_{5}=s_{4}+\frac{R_{56}^{(2)}}{2} \delta_{4}=\frac{s_{0}}{C_{1}}+\frac{h_{11} R_{66} R_{56}^{(2)}}{2} s_{0}+\frac{h_{21} R_{56}^{(2)}}{2 C_{1}} s_{0}$

After the second octupole, the transverse coordinates in the bend plane of the chicane, $\left(x_{5}, x_{5}^{\prime}\right)$, are given in terms of the dispersion at the octupole entrance, $\eta_{2}$, the $\mathrm{BC} 1$ octupole kick after transport through an accelerating section with $n \pi$ betatron phase advance, and the second octupole kick:

$$
\begin{gathered}
x_{5}=\eta_{2} \delta_{4}=\eta_{2}\left(h_{11} R_{66} s_{0}+\frac{h_{21}}{C_{1}} s_{0}\right) \\
x_{5}^{\prime}=(-1)^{n} \sqrt{\frac{\beta_{1} E_{1}}{\beta_{2} E_{2}}} x_{2}^{\prime}+\frac{1}{6} K_{3}^{(2)} L_{2} x_{5}^{3} .
\end{gathered}
$$

At the $\mathrm{BC} 2$ chicane exit, the longitudinal phase space coordinates can be expressed in terms of the total linear compression factor, $\mathrm{C}_{2}$ :

$$
\begin{aligned}
\delta_{6} & =h_{11} R_{66} s_{0}+\frac{h_{21}}{C_{1}} s_{0} \\
s_{6} & =s_{4}+R_{56}^{(2)} \delta_{4}=\frac{s_{0}}{C_{1}}+h_{11} R_{66} R_{56}^{(2)} s_{0}+\frac{h_{21} R_{56}^{(2)}}{C_{1}} s_{0} \\
& =\frac{s_{0}}{C_{2}} C_{2}=\frac{C_{1}}{1+C_{1} h_{11} R_{66} R_{56}^{(2)}+h_{21} R_{56}^{(2)}} .
\end{aligned}
$$

It is convenient for our purposes to express the octupole kicks in terms of the $\mathrm{BC} 1$ and $\mathrm{BC} 2$ chicane parameters and linear compression factors. With this in mind, the initial chirp can be expressed in terms of the BC1 linear compression factor and $R_{56}$ :

$$
h_{11}=\frac{1-C_{1}}{C_{1} R_{56}^{(1)}} .
$$

The transverse offset and longitudinal position at the $\mathrm{BC} 1$ octupole are given by:

$$
\begin{aligned}
& x_{2}=\eta_{1} \delta_{1}=\eta_{1} \frac{1-C_{1}}{C_{1} R_{56}^{(1)}} s_{0} \\
& s_{2}=s_{0}\left(1+\frac{1}{2} h_{11} R_{56}^{(1)}\right)=s_{0} \frac{1+C_{1}}{2 C_{1}} .
\end{aligned}
$$

In order to compare with simulations, we write $x_{2}$ in terms of the compressed beam coordinate, $s_{2}$ with $\eta_{1}=$ $\theta_{1}\left(l_{d 1}+l_{b 1}\right)$ and $R_{56}^{(1)}=-2 \theta_{1}^{2}\left(l_{d 1}+\frac{2}{3} l_{b 1}\right)$ :

$$
\begin{aligned}
x_{2} & =\eta_{1} \frac{1-C_{1}}{C_{1} R_{56}^{(1)}} \frac{2 C_{1}}{1+C_{1}} s_{2}=\frac{2 \eta_{1}}{R_{56}^{(1)}} \frac{1-C_{1}}{1+C_{1}} s_{2} \\
& =\frac{1}{\theta_{1}} \frac{l_{d 1}+l_{b 1}}{l_{d 1}+\frac{2}{3} l_{b 1}} \frac{C_{1}-1}{1+C_{1}} s_{2} .
\end{aligned}
$$

The $\mathrm{BC} 1$ octupole kick is given by: 


$$
\begin{aligned}
& x_{2}^{\prime}=\frac{1}{6} K_{3}^{(1)} L_{1} x_{2}{ }^{3} \\
& x_{2}^{\prime}=\frac{1}{6} K_{3}^{(1)} L_{1}\left(\frac{l_{d 1}+l_{b 1}}{l_{d 1}+\frac{2}{3} l_{b 1}}\right)^{3} \frac{1}{\theta_{1}{ }^{3}}\left(\frac{C_{1}-1}{C_{1}+1}\right)^{3} s_{2}{ }^{3} .
\end{aligned}
$$

This is Eq. (10) as given in the main text. Similarly we write the chirp at $\mathrm{BC} 2$ in terms of the $\mathrm{BC} 1$ linear compression factor and total compression factor, from Eq. (C8) and (C9):

$$
\begin{aligned}
h_{21} & =\frac{C_{1}-C_{2}}{C_{2} R_{56}^{(2)}}-\frac{\left(1-C_{1}\right) R_{66}}{R_{56}^{(1)}} \\
\delta_{4} & =s_{0} \frac{C_{1}-C_{2}}{C_{1} C_{2} R_{56}^{(2)}} .
\end{aligned}
$$

The transverse offset and longitudinal position at the $\mathrm{BC} 2$ octupole are given by:

$$
\begin{aligned}
& x_{5}=\eta_{2} \delta_{4}=\eta_{2} s_{0} \frac{C_{1}-C_{2}}{C_{1} C_{2} R_{56}^{(2)}} \\
& s_{5}=\frac{s_{0}}{C_{1}}+\frac{R_{56}^{(2)}}{2} \delta_{4}=s_{0} \frac{C_{2}+C_{1}}{2 C_{1} C_{2}} .
\end{aligned}
$$

Again we write $x_{5}$ in terms of the compressed beam coordinate, $s_{5}$, with $\eta_{2}=\theta_{2}\left(l_{d 2}+l_{b 2}\right)$ and $R_{56}^{(2)}=$ $-2 \theta_{2}^{2}\left(l_{d 2}+\frac{2}{3} l_{b 2}\right)$ :

$$
x_{5}=\frac{2 \eta_{2}}{R_{56}^{(2)}} \frac{C_{1}-C_{2}}{C_{2}+C_{1}} s_{5}=\frac{1}{\theta_{2}} \frac{l_{d 2}+l_{b 2}}{l_{d 2}+\frac{2}{3} l_{b 2}} \frac{C_{2}-C_{1}}{C_{2}+C_{1}} s_{5} .
$$

The kick from the second octupole, $x_{5(2)}^{\prime}$, is given by:

$$
x_{5(2)}^{\prime}=\frac{1}{6} K_{3}^{(2)} L_{2} \frac{1}{\theta_{2}^{3}}\left(\frac{l_{d 2}+l_{b 2}}{l_{d 2}+\frac{2}{3} l_{b 2}}\right)^{3}\left(\frac{C_{2}-C_{1}}{C_{2}+C_{1}}\right)^{3} s_{5}^{3} .
$$

This is Eq (12) as given in the main text. To express the first octupole kick transported to the second octupole we write $x_{2}$ in terms of the compressed beam coordinate, $s_{5}$ :

$$
x_{2}=\frac{2 \eta_{1}}{R_{56}^{(1)}} \frac{C_{2}\left(1-C_{1}\right)}{C_{2}+C_{1}} s_{5}=\frac{1}{\theta_{1}} \frac{l_{d 1}+l_{b 1}}{l_{d 1}+\frac{2}{3} l_{b 1}} \frac{C_{2}\left(C_{1}-1\right)}{C_{2}+C_{1}} s_{5} .
$$

The contribution from the first octupole, $x_{5(1)}^{\prime}$, is given by:

$$
\begin{aligned}
x_{5(1)}^{\prime}= & (-1)^{n} \sqrt{\frac{\beta_{1} E_{1}}{\beta_{2} E_{2}}} \frac{1}{6} K_{3}^{(1)} L_{1} x_{2}{ }^{3} \\
x_{5(1)}^{\prime}= & (-1)^{n} \sqrt{\frac{\beta_{1} E_{1}}{\beta_{2} E_{2}}} \frac{1}{6} K_{3}^{(1)} L_{1} \\
& \times \frac{1}{\theta_{1}^{3}}\left(\frac{l_{d 1}+l_{b 1}}{l_{d 1}+\frac{2}{3} l_{b 1}}\right)^{3}\left(\frac{C_{2}\left(C_{1}-1\right)}{C_{2}+C_{1}}\right)^{3} s_{5}{ }^{3} .
\end{aligned}
$$

This is Eq (11) as given in the main text. The net octupole kick after the second octupole can now be written as the sum of Eq. (C16) and Eq. (C18). By setting this net kick to zero, the relationship of the two octupole strengths can be achieved as shown in Eq. (13).

[1] P. Neyman, W. B. Colson, S. C. Gottshalk, A. M. M. Todd, J. Blau, and K. Cohn, Free electron lasers in 2017, in Proceedings of the 38th International Free Electron Laser Conference (FEL17), Santa Fe, NM, 2017 (JACoW, Geneva, 2018), pp. 204209.

[2] H. Wiedemann, Particle Accelerator Physics, 3rd ed. (SpringerVerlag, Berlin, 2007).

[3] M. Venturini, Models of longitudinal space-charge impedance for microbunching instability, Phys. Rev. Accel. Beams 11, 034401 (2008).

[4] J. B. Murphy, An Introduction to Coherent Synchrotron Radiation in Storage Rings, ICFA Beam Dynamics Newsletter No. 35, p. 20-27 (2004).

[5] K. L.F. Bane et al., Measurements and modeling of coherent synchrotron radiation and its impact on the Linac Coherent Light Source electron beam, Phys. Rev. Accel. Beams 12, 030704 (2009).

[6] K.F. Bane, Wakefields of sub-picosecond electron bunches, Report No. SLAC-PUB-11829, 2006.

[7] A. Novokhatski and A. Mosnier, Short bunch wake potentials for a chain of TESLA cavities, Nucl. Instrum. Methods Phys. Res., Sect. A 763, 202 (2014).

[8] P. Emma, X-Band RF harmonic compensation for linear bunch compression in the LCLS, LCLS technical note, Report No. SLAC-TN-05-004, 2001.

[9] Y. Sun, P. Emma, T. Raubenheimer, and J. Wu, X-band rf driven free electron laser driver with optics linearization, Phys. Rev. Accel. Beams 17, 110703 (2014).

[10] S. Thorin et al., Experience and initial measurements of magnetic linearisation in the MAX IV linac bunch compressors, Proc. of 38th Free Electron Laser Conf., FEL 2017, Santa Fe, NM, USA, TUP013 (JACoW, Geneva, 2017).

[11] S. Di Mitri, M. Cornacchia, and S. Spampinati, Cancellation of Coherent Synchrotron Radiation Kicks with Optics Balance, Phys. Rev. Lett. 110, 014801 (2013).

[12] C. Mitchell, J. Qiang, and P. Emma, Longitudinal pulse shaping for the suppression of coherent synchrotron radiation-induced emittance growth, Phys. Rev. Accel. Beams, 16, 060703 (2013).

[13] G. Penco, M. Danailov, A. Demidovich, E. Allaria, G. De Ninno, S. Di Mitri, W. M. Fawley, E. Ferrari, L. Giannessi, 
and M. Trovó, Experimental Demonstration of Electron Longitudinal-Phase-Space Linearization by Shaping the Photoinjector Laser Pulse, Phys. Rev. Lett. 112, 044801 (2014).

[14] G. Penco et al., Passive Linearization of the Magnetic Bunch Compression Using Self-Induced Fields, Phys. Rev. Lett. 119, 184802 (2017).

[15] H.X. Deng et al., Experimental Demonstration of Longitudinal Beam Phase-Space Linearizer in a Free-Electron Laser Facility by Corrugated Structures, Phys. Rev. Lett. 113, 254802 (2014).

[16] Y. Ding et al., Beam shaping with a passive linearizer at the LCLS-II for high-current operation, Report No. LCLS-IITN-18-02, 2018.

[17] J. Arther et al., Linac Coherent Light Source (LCLS) Conceptual Design Report No. SLAC-R-593, 2002.

[18] B. Beutner, Longitudinal design and RF stability requirements for the SwissFEL facility, Proc. of the 4th International Particle Accelerator Conf., IPAC 2013, Shanghai, China (JACoW, Geneva, 2013), WEPFI057, p. 281.

[19] T. K. Charles, J. B. Svensson, A. Latina, and S. Thorin, First experimental measurements of the caustic nature of trajectories in bunch compressors, Proc. of the 10th International Particle Accelerator Conf., IPAC 2019, Melbourne, Australia (JACoW, Geneva, 2019), WEYYPLS2.

[20] S. Di Mitri and M. Cornacchia, Electron beam brightness in linac drivers for free-electron-lasers, Phys. Rep. 539, 1 (2014).

[21] T. Raubenheimer et al., LCLS-II final design report. Technical report, SLAC Technical Report. No. LCLSII1. 1-DR-0251-R0, 2015.

[22] Y. Ding et al., Beam shaping to improve the free-electron laser performance at the Linac Coherent Light Source, Phys. Rev. Accel. Beams 19, 100703 (2016).
[23] T. K. Charles, D. M. Paganin, A. Latina, M. J. Boland, and R. T. Dowd, Current-horn suppression for reduced coherent-synchrotron-radiation-induced emittance growth in strong bunch compression, Phys. Rev. Accel. Beams, 20, 030705 (2017).

[24] T. K. Charles, D. M. Paganin, and R. T. Dowd, Causticbased approach to understanding bunching dynamics and current spike formation in particle bunches, Phys. Rev. Accel. Beams, 19, 104402 (2016).

[25] Y. Ding, K. Bane, and Y. Nosochkov, Beam shaping for high-repetition-rate X-ray FELs, Proc. of 39th Free Electron Laser Conf., FEL 2019, Hamburg, Germany (JACoW, Geneva, 2019), THP035

[26] M. Borland, ELEGANT: A flexible SDDS-compliant code for accelerator simulation, ANL Advanced Photon Source Report No. LS-287, 2000.

[27] A. W. Chao and M. Tigner, Handbook of Accelerator Physics and Engineering (World Scientific, Singapore, 1999).

[28] J. Qiang, R. D. Ryne, S. Habib, and V. Decyk, An objectoriented parallel particle-in-cell code for beam dynamics simulation in linear accelerators, J. Comput. Phys. 163, 434 (2000).

[29] J. Qiang, S. Lidia, R. D. Ryne, and C. Limborg-Deprey, Three-dimensional quasistatic model for high brightness beam dynamics simulation, Phys. Rev. Accel. Beams 9, 044204 (2006).

[30] Y. Nosochkov, Compensation of emittance growth from octupoles in BC1 and BC2 chicanes, Report No. LCLS-IITN-20-04, 2020.

[31] N. Sudar, K. Bane, Y. Ding, Y. Nosochkov, and Z. Zhang, Non-linear compression with octupoles for current profile shaping, Report No. LCLS-II-TN-20-05 2020. 